\title{
OBESITY
}

\section{New strategy for diet-induced obesity}

A paper recently published in Nature Communications reports the design of a closed-loop genetic circuit that monitors blood levels of fatty acids and coordinates the expression of pramlintide (a synthetic analogue of islet amyloid polypeptide that suppresses appetite) in mice.

"We urgently need new treatment strategies for obesity as the global drug pipelines are dry," states corresponding author Martin Fussenegger. Synthetic biology has now advanced enough to enable the design of closed-loop networks that can successfully interface with mammalian physiology. These advances led Fussenegger and co-workers to apply this strategy to the treatment of diet-induced obesity.

The researchers designed a synthetic lipid sensor that acts as a transcription factor by grafting the human PPARa (which manages the mobilisation of lipids from fat stores) onto the repressor TtgR. In the absence of lipids, the lipid sensor suppresses expression of pramlintide. Engineered cells containing this sensor were encapsulated in alginate microcontainers and implanted into wildtype mice. The mice were fed an unlimited low, medium or high-fat diet.

"The sensor-effector device detects a range of dietary lipids and converts their presence into a dose-dependent expression of the appetite-reducing pramlintide," explains Fussenegger. Implanted mice had reduced food consumption, blood lipid levels and body weight when on a medium or high-fat diet; levels were unchanged in mice fed a low-fat diet.

"A closed-loop control circuit interfacing fatty acid metabolism and controlling dietary intake has never been done before," says Fussenegger. The team are now hoping to conduct preclinical trials in order to apply this treatment strategy in patients.

\section{Claire Greenhill}

Original article Rössger, K. et al. A closed-loop synthetic gene circuit for the treatment of diet-induced obesity in mice. Nat. Commun. doi:10.1038/ncomms3825 